\title{
The antitumor function of arctigenin in human retinoblastoma cells is mediated by jagged-1
}

\author{
NING KE, QING LIU, LIANHONG PI, JING FANG, LIN CHEN and XINKE CHEN \\ Department of Ophthalmology, Children's Hospital, Chongqing Medical University, Chongqing 400014, P.R. China
}

Received June 10, 2018; Accepted February 19, 2019

DOI: $10.3892 / \mathrm{mmr} .2019 .10026$

\begin{abstract}
Retinoblastoma is an intraocular malignant tumor that may severely affect vision and represents a life-threatening disease in children. Arctigenin (ATG) is an active compound that exhibits numerous pharmacological activities, which is isolated from the seeds of greater burdock (Arctium lappa Linnaeus), a plant used in traditional Chinese herbal medicine. The present study aimed to investigate the effects of ATG on cancer progression by analyzing the retinoblastoma cell line Y79. ATG exhibited a significant inhibitory effect on the viability of Y79 cells in a dose-dependent manner. Furthermore, treatment with ATG promoted apoptosis, and increased the protein expression levels of B-cell lymphoma 2 (BCL-2)-associated $\mathrm{X}$ protein and decreased the protein expression levels of BCL-2. Cell migration was suppressed following treatment with ATG, as assessed by Transwell migration assay. Furthermore, the protein expression levels of jagged-1 (JAG1) were decreased, and various factors involved in the Notch signaling pathway, including the Notch intracellular domain (NICD), transcription factor HES (HES)5 and HES1 were downregulated following treatment with ATG. The decreased expression levels of JAG1 were restored in response to JAG1 overexpression, alongside increases in the protein expression levels of NICD, HES5 and HES1. Furthermore, overexpression of JAG1 partly restored the cell viability and migration suppressed following treatment with ATG. In addition, ATG-induced apoptosis was reduced by JAG1 overexpression. Collectively, the present results suggested that ATG may serve as an antitumor compound by suppressing the proliferation and migration of retinoblastoma cells, inducing apoptosis, downregulating the protein expression levels of JAG1, and decreasing the activity of the Notch signaling pathway.
\end{abstract}

Correspondence to: Professor Qing Liu, Department of Ophthalmology, Children's Hospital, Chongqing Medical University, 136 Second Zhongshan Road, Chongqing 400014, P.R. China E-mail: liuq0823_1@163.com

Key words: arctigenin, retinoblastoma, jagged-1, Notch signaling pathway, antitumor

\section{Introduction}

Retinoblastoma is an intraocular malignant tumor derived from immature retinal cells (1). This malignancy is commonly observed in children $<3$ years old and poses a severe threat to vision and life. The intraocular proliferation and migration of retinoblastoma cells may lead to the necrosis of retinal tissues and subsequent retinal detachment, in addition to the development of intracranial metastasis and metastasis to the optic nerve (2). To the best of our knowledge, the current strategies available for retinoblastoma treatment are limited, and include surgical resection, radiotherapy and systemic chemotherapy (3). Although the 5-year survival rate of early diagnosed patients has improved to $>90 \%$ over recent years (4), the side effects of the currently available therapies include facial defects, optic nerve injury, visual disorders and drug toxicity. Therefore, novel treatments and therapeutic strategies are required to improve the outcomes of patients with retinoblastoma.

Arctigenin (ATG) is an active compound extracted from the seeds of greater burdock (Arctium lappa Linnaeus), a plant used in traditional Chinese herbal medicine. ATG has been demonstrated to exhibit pharmacological activities in the treatment of diabetes (5), obesity (6) and inflammation (7). In addition, in vitro and in vivo studies demonstrated that ATG possesses antiproliferative, proapoptotic, antimetastatic and drug-resistance-decreasing effects in various types of cancer by influencing the activity of numerous signaling pathways $(8,9)$ and molecular markers $(10,11)$. However, the effects of ATG on the biological progression of retinoblastoma remain unclear.

The Notch signaling pathway mediates signal transduction between adjacent cells and serves an important role in cancer progression $(12,13)$. Dysregulation of the Notch signaling pathway was observed in various types of cancer $(14,15)$. In mammals there are five main ligands: Jagged (JAG)1, JAG2, and $\delta$-like canonical Notch ligands 1,3 and 4 . The interaction between one ligand and one of the four Notch receptors (NOTCH1-4) activates cleavage of the receptor (13). Following proteolytic cleavage, the Notch intracellular domain (NICD) is released from the cell membrane and enters the nucleus to activate transcription of its downstream genes (13). JAG1 is an important Notch ligand and is able to promote activation of the Notch signaling pathway, serving as an oncogene in certain types of cancer (16). The present study aimed to investigate the effects of ATG on retinoblastoma and its underlying molecular 
mechanism by examining the involvement of the JAG1-Notch signaling pathway.

\section{Materials and methods}

Reagents and cell culture. ATG ( $\geq 95 \%$ purity) was purchased from Sigma-Aldrich (Merck KGaA, Darmstadt, Germany), dissolved in dimethyl sulfoxide (DMSO) at a concentration of $50 \mathrm{mM}$ and stored at $-20^{\circ} \mathrm{C}$. The human retinoblastoma cell line Y79 was purchased form The Cell Bank of Type Culture Collection of Chinese Academy of Science (Shanghai, China) and cultivated in RPMI-1640 medium (Gibco; Thermo Fisher Scientific, Inc., Waltham, MA, USA) supplemented with $20 \%$ fetal bovine serum (FBS) (Gibco; Thermo Fisher Scientific, Inc.), streptomycin $(100 \mathrm{U} / \mathrm{ml})$ and penicillin $(100 \mathrm{U} / \mathrm{ml})$ at $37^{\circ} \mathrm{C}$ in a humidified atmosphere containing $5 \% \mathrm{CO}_{2}$.

Plasmid construction and transfection. TRIzol ${ }^{\circledR}$ reagent (Invitrogen; Thermo Fisher Scientific, Inc.) was used to isolate RNA from Y79 cells. Subsequently, cDNA was synthesized from RNA using the PrimeScript ${ }^{\mathrm{TM}}$ reverse transcription (RT) reagent kit with genomic DNA Eraser (Takara Biotechnology Co., Ltd., Dalian, China) according to the manufacturer's protocol. The forward (5'-CCCAAGCTTATGCGTTCC CCACGGACGC-3') and reverse (5'-CCGGAATTCCTA TACGATGTACTCCATTCGGTTTAAGCTC-3') primers were designed for cloning the coding sequence of JAG1 using the cDNA extracted from Y79 cells as a template. The polymerase chain reaction (PCR) was performed using the PrimeSTAR HS DNA polymerase (Takara Biotechnology Co., Ltd.) with the conditions as follows: Initial denaturation at $94^{\circ} \mathrm{C}$ for $10 \mathrm{~min}$ followed by 30 cycles each consisting of $98^{\circ} \mathrm{C}$ for $20 \mathrm{sec}, 50^{\circ} \mathrm{C}$ for $20 \mathrm{sec}$, and $72^{\circ} \mathrm{C}$ for $5 \mathrm{~min}$ and a final extension at $72^{\circ} \mathrm{C}$ for $10 \mathrm{~min}$. The obtained DNA was subsequently cloned into a pcDNA3.1(+) plasmid (Invitrogen; Thermo Fisher Scientific, Inc.). The generated recombinant plasmid pcDNA-JAG1 was sequenced by Sangon Biotech Co. Ltd. (Shanghai, China). Cells $\left(2 \times 10^{5} /\right.$ well) was transfected with 500 ng plasmid using Lipofectamine ${ }^{\circledR} 2000$ (Invitrogen; Thermo Fisher Scientific, Inc.) according to the manufacturer's protocol. Following incubation for $48 \mathrm{~h}$, cells were harvested for further experimentation

Reverse transcription-quantitative $(R T-q)$ PCR. TRIzol ${ }^{\circledR}$ reagent (Invitrogen; Thermo Fisher Scientific, Inc.) was used to isolate RNA from Y79 cells. cDNA was synthesized from RNA using the PrimeScript ${ }^{\mathrm{TM}}$ RT reagent kit with genomic DNA Eraser (Takara Biotechnology Co., Ltd.) according to the manufacturer's protocol. qPCR was conducted using a SYBR Green kit (Takara Biotechnology Co., Ltd.) and the 7500 Real-Time PCR System (Applied Biosystems; Thermo Fisher Scientific, Inc.). Primers used were: JAG1, forward, 5'-GGGGCAACA CCTTCAACCTC-3' and reverse, 5'-CCACGCCTCCACAAG CAAC-3'; GADPH was set as internal control forward, 5'-GCG ACACCCACTCCTCCAC-3' and reverse, 5'-TCCACCACC CTGTTGCTGTAG-3'). Thermocycling conditions were as follows: Initial denaturation at $95^{\circ} \mathrm{C}$ for $30 \mathrm{sec}$ followed by 40 cycles each consisting of $95^{\circ} \mathrm{C}$ for $5 \mathrm{sec}$ and $60^{\circ} \mathrm{C}$ for $40 \mathrm{sec}$. The relative changes in the expression of target genes were calculated by $2^{-\Delta \Delta \mathrm{Cq}}$ method (17).
Cell viability and proliferation assays. Cells were seeded into a 96-well plate at a density of $3 \times 10^{3}$ cells/well. After a 24-h incubation at $37^{\circ} \mathrm{C}$, the medium was replaced with fresh medium containing ATG or the equivalent volume of DMSO at $37^{\circ} \mathrm{C}$ for $96 \mathrm{~h}$. Cell viability was subsequently measured using the Cell Counting kit-8 (CCK-8; Beyotime Institute of Biotechnology, Haimen, China) according to the manufacturer's protocol.

For the 5-ethynyl-2'-deoxyuridine (EdU) incorporation assay, floating cells $\left(1 \times 10^{4} /\right.$ well $)$ were plated on poly-L-lysine-coated microscope slides. Subsequently, cell proliferation was measured using an EdU kit (Guangzhou RiboBio Co., Ltd., Guangzhou, China) according to the manufacturer's protocol. Images were obtained using a Fluoview1000 laser scanning confocal microscope (Olympus Corporation, Tokyo, Japan). Cell counting was performed using ImageJ version 1.8.0 software (National Institutes of Health, Bethesda, MD, USA).

Apoptosis assay. The treated cells were collected and washed twice with ice-cold PBS. The apoptotic rate was measured using a fluorescein isothiocyanate-labeled Annexin V apoptosis detection kit according to the manufacturer's protocol, and was quantified using an Accuri C6 flow cytometer and CFlow plus version 1.0.264.15 software (all BD Biosciences, Franklin Lakes, NJ, USA).

Migration assay. Polycarbonate membrane inserts with $8-\mu \mathrm{m}$ pores and Transwell plates (Corning Inc., Corning, NY, USA) were used for the cell migration assay. After a 24-h incubation, cells were cultured in serum-free medium containing hydroxyurea $(1.8 \mathrm{mM})$ for $12 \mathrm{~h}$ at $37^{\circ} \mathrm{C}$ to synchronize cells and suppress cell proliferation. Subsequently, cells were trypsinized and reseeded in serum-free RPMI-1640 medium [containing 10\% bovine serum albumin (BSA) (Gibco; Thermo Fisher Scientific, Inc.) and ATG or the equivalent volume of DMSO] and transferred to the upper chamber at a density of $5 \times 10^{4}$ cells/well. RPMI-1640 medium supplemented with $20 \%$ FBS was subsequently added into the lower chamber. After a 48 -h incubation at $37^{\circ} \mathrm{C}$, the migratory cells were fixed with methanol at room temperature for $30 \mathrm{~min}$ and stained using $0.1 \%$ crystal violet at room temperature for $20 \mathrm{~min}$. The migrated cells were counted in five randomly selected fields of view under an inverted light microscope.

Western blot analysis. The cells were collected, washed twice with PBS and lysed with radioimmunoprecipitation assay lysis buffer (Beyotime Institute of Biotechnology) for total protein extraction. The protein concentration was determined using a bicinchoninic acid assay kit (Beyotime Institute of Biotechnology). Equal amounts of protein (50 $\mu \mathrm{g} /$ lane) were subsequently separated by $10 \%$ SDS-PAGE and transferred onto a polyvinylidene difluoride membrane. After blocking with BSA for $1 \mathrm{~h}$ at room temperature, the membranes were incubated with primary antibodies purchased from Abcam (Cambridge, UK). The primary antibodies used were as follows: Anti-JAG1 (cat. no. ab7771; 1:500), anti-NICD (cat. no. ab8925; 1:500), anti-transcription factor HES (HES)1 (cat. no. ab71559; 1:1,000), anti-HES5 (cat. no. ab25374, 1:1,000), anti-B-cell lymphoma 2 (BCL2; cat. no. ab32124; 1:1,000), anti-BCL2-associated X 
A

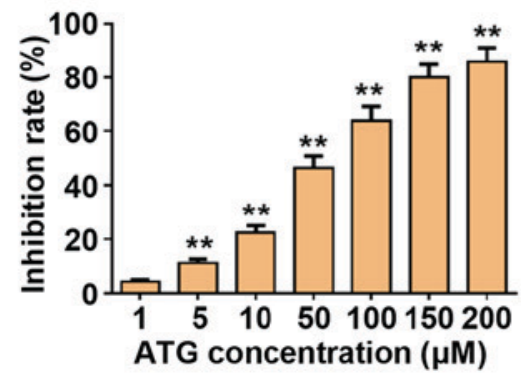

B

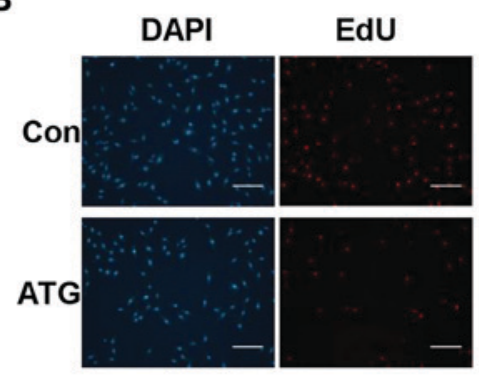

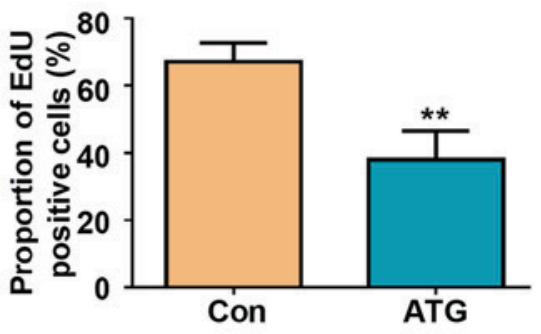

Figure 1. ATG inhibits viability of the retinoblastoma cell line Y79. (A) Viability of Y79 cells treated with various concentrations of ATG was assessed by Cell Counting kit-8 assay. (B) Effects of ATG $(50 \mu \mathrm{M})$ on cell proliferation was determined using EdU staining. Cells treated with an equivalent volume of dimethyl sulfoxide were used as a negative control. Scale bar, $100 \mu \mathrm{m}$. ${ }^{* *} \mathrm{P}<0.01$ vs. Con. ATG, arctigenin; Con, control; EdU, 5-ethynyl-2'-deoxyuridine.

protein (BAX; cat. no. ab32503; $1: 2,000)$ and anti- $\beta$-actin (cat. no. ab8227; 1:2,000). The primary antibodies were incubated overnight at $4^{\circ} \mathrm{C}$. The membranes were then incubated with a horseradish peroxidase-conjugated secondary antibody for $1 \mathrm{~h}$ at room temperature (cat. no. ab205718; 1:2,000; Abcam) and the bands were developed using the Ultrasensitive enhanced chemiluminescence kit (Sangon Biotech Co., Ltd.). Protein quantification were performed by ImageJ version 1.8.0 software (National Institutes of Health).

Immunofluorescence assay. Treated cells were collected by centrifugation in $300 \mathrm{x}$ g at $4^{\circ} \mathrm{C}$ for $5 \mathrm{~min}$ and the pellet was washed twice with PBS. Subsequently, the cells were fixed with $4 \%$ paraformaldehyde for at room temperature $20 \mathrm{~min}$, washed twice with PBS, and treated with $0.5 \%$ Triton X-100 for $5 \mathrm{~min}$. The cells were washed twice with PBS and blocked using 5\% BSA at room temperature for $1 \mathrm{~h}$. After blocking, the cells were incubated with a primary antibody against JAG1 (cat. no. ab7771; 1:300; Abcam) at $37^{\circ} \mathrm{C}$ for $1 \mathrm{~h}$ and rinsed in PBS. Subsequently, the cells were incubated with an Alexa Fluor ${ }^{\circledR}$ 647-conjugated secondary antibody (cat. no. ab150075; 1:500; Abcam) at $37^{\circ} \mathrm{C}$ for $1 \mathrm{~h}$. Cells were placed onto slides and mounted using Fluoromount- $\mathrm{G}^{\mathrm{TM}}$ with DAPI (Thermo Fisher Scientific, Inc.). The images were obtained using a laser scanning confocal microscope (Olympus Corporation).

Statistical analysis. Statistical analysis was performed using SPSS 17.0 (SPSS, Inc., Chicago, IL, USA). The data are presented as the means \pm standard deviation of at least three independent experiments. Statistical differences were analyzed by Student's t-test or one-way analysis of variance followed by Student-Newman-Keuls post hoc test for multiple comparisons. $\mathrm{P}<0.05$ was considered to indicate a statistically significant difference.

\section{Results}

Effects of ATG on the viability of retinoblastoma cells. To investigate the effects of ATG on cell viability, the retinoblastoma cell line Y79 was treated with various concentrations of ATG for $48 \mathrm{~h}$. ATG significantly decreased cell viability in a dose-dependent manner, as detected by CCK-8 assay (Fig. 1A). To further assess the effects of ATG on cell proliferation, an EdU labeling assay was performed following treatment with $50 \mu \mathrm{M}$ ATG, a concentration identified to exhibit an inhibition rate of $46.5 \%$, as assessed by CCK-8 assay (Fig. 1A). Treatment with ATG significantly decreased the number of EdU-positive cells from 67.9 to $39.4 \%$ (Fig. 1B). The present results suggested that ATG may inhibit the viability and proliferation of Y79 retinoblastoma cells.

Effects of ATG on apoptosis of human retinoblastoma cells. Apoptosis was analyzed following treatment with ATG by performing an Annexin V/propidium iodide assay. The proportion of cells in the early and late stages of apoptosis was increased by 114 and 468\%, respectively (Fig. 2A). Furthermore, western blotting assessed the protein expression levels of the apoptosis-associated factors BCL2 and BAX. Treatment with ATG increased the protein expression levels of the pro-apoptotic factor BAX and decreased the protein expression levels of the anti-apoptotic protein BCL2 (Fig. 2B). The present results suggested that treatment with ATG induced apoptosis of Y79 retinoblastoma cells.

Effects of ATG on retinoblastoma cell migration. The effects of ATG were also investigated on retinoblastoma cell migration using a Transwell migration assay. The average number of migrated cells was significantly decreased by $66.7 \%$ following treatment with ATG (Fig. 3). The present results suggested that treatment with ATG inhibited the migration of Y79 retinoblastoma cells.

Effects of ATG on the Notch signaling pathway in human retinoblastoma cells. The Notch signaling pathway serves an important role in cell proliferation, migration and apoptosis. To investigate the molecular mechanism underlying the anticarcinogenic effects of ATG, the effects of ATG on JAG1 protein expression were analyzed by immunofluorescence (Fig. 4A) and western blotting (Fig. 4B). The protein expression levels of JAG1 were markedly decreased following treatment with ATG compared with in the control group (Fig. 4A-C). Additionally, the protein expression levels of various factors involved in the Notch signaling pathway, including NICD, HES1 and HES5 were significantly decreased following treatment with ATG, as assessed by western blotting (Fig. 4B and C). The present results suggested that ATG suppressed the protein expression 
A
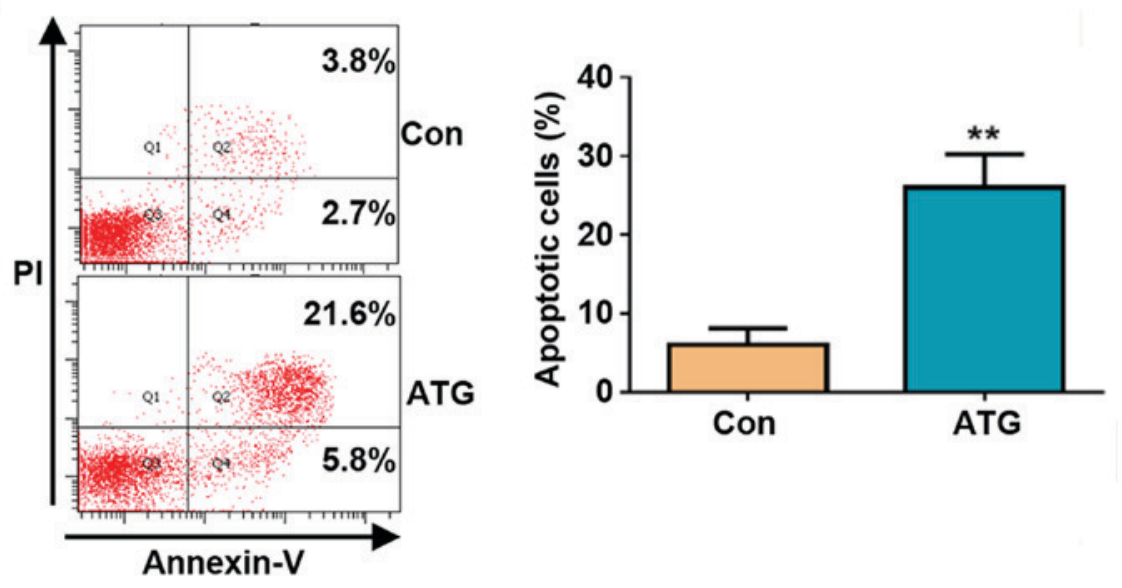

B
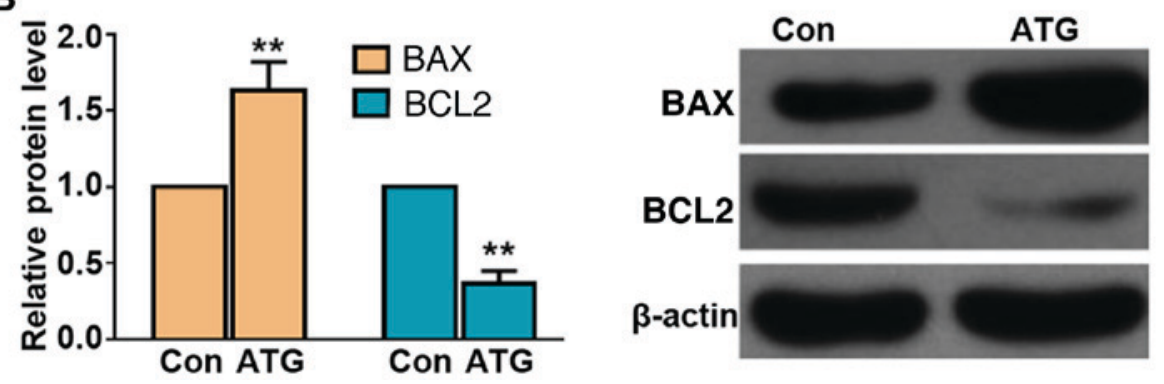

Figure 2. ATG induces apoptosis of the retinoblastoma cell line Y79. (A) Apoptosis was determined by Annexin V/PI staining. (B) Protein expression levels of apoptosis-associated factors were analyzed by western blotting. Cells were treated with ATG $(50 \mu \mathrm{M})$ or an equivalent volume of dimethyl sulfoxide. ${ }^{* *} \mathrm{P}<0.01$ vs. Con. ATG, arctigenin; BAX, BCL2-associated X protein; BCL2, B-cell lymphoma 2; Con, control; PI, propidium iodide.
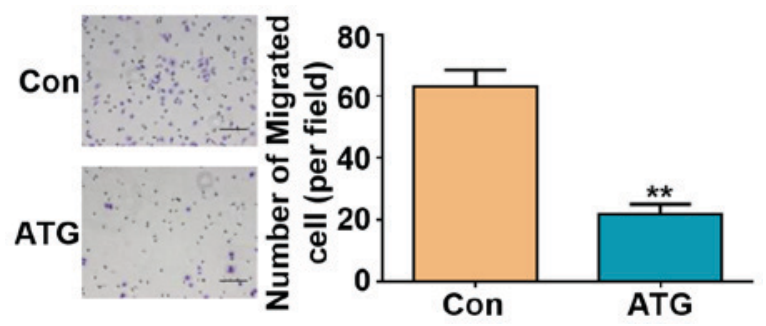

Figure 3. ATG inhibits cell migration of the retinoblastoma cell line Y79. Cells were treated with ATG $(50 \mu \mathrm{M})$ or an equivalent volume of dimethyl sulfoxide. Scale bar, $100 \mu \mathrm{m} .{ }^{* *} \mathrm{P}<0.01$ vs. Con. ATG, arctigenin; Con, control.

levels of JAG1 and inhibited the Notch signaling pathway in Y79 retinoblastoma cells.

JAG1 mediates the antitumor effects of ATG in human retinoblastoma cells. To examine the role of JAG1 in the anticarcinogenic effects of ATG on retinoblastoma, JAG1 was overexpressed by transfecting Y79 cells with pCDNA-JAG1. The mRNA expression levels of JAG1 were significantly upregulated in the transfected cells, as measured by RT-quantitative polymerase chain reaction (Fig. 5A). The immunofluorescence and western blotting results further suggested that transfection with the recombinant plasmid significantly increased the expression levels of JAG1 following treatment with ATG (Fig. 5B and 5C). The protein expression levels of NICD, HES1 and HES5 were also significantly increased (Fig. 5D and 5E). Furthermore, JAG1 overexpression significantly reversed the cell viability suppressed by treatment with ATG, as determined using the CCK-8 assay (Fig. 5F). The migratory ability of retinoblastoma cells was also partly restored by overexpressing JAG1 (Fig. 6A). Furthermore, overexpression of JAG1 partially abrogated the pro-apoptotic effects of ATG (Fig. 6B). The present results suggested that ATG may serve as an antitumor compound partly by downregulating the protein expression levels of JAG1 in Y79 retinoblastoma cells.

\section{Discussion}

In a previous study, ATG exhibited increased antitumor activity against retinoblastoma cells compared with carboplatin in vitro $(18,19)$, indicating its potential clinical value in the treatment of retinoblastoma; however, ATG does not represent a current available therapy to treat patients with retinoblastoma, to the best of our knowledge. The antitumor potential of ATG has been demonstrated in various types of cancer in vitro and in vivo. In the HepG2 liver cancer cell line, ATG was revealed to inhibit cell proliferation and colony formation by recruiting CCAAT-enhancer binding protein $\alpha$ and peroxisome proliferator-activated receptor $\alpha$ to the promoter of ankyrin, downregulating its expression levels (20). In addition, ATG decreases cell proliferation and promotes apoptosis of the $\mathrm{H} 460$ non-small lung cancer cell line by suppressing the expression level of surviving (10). Similarly, treatment with ATG induces cell senescence and inhibits tumor growth of gallbladder 

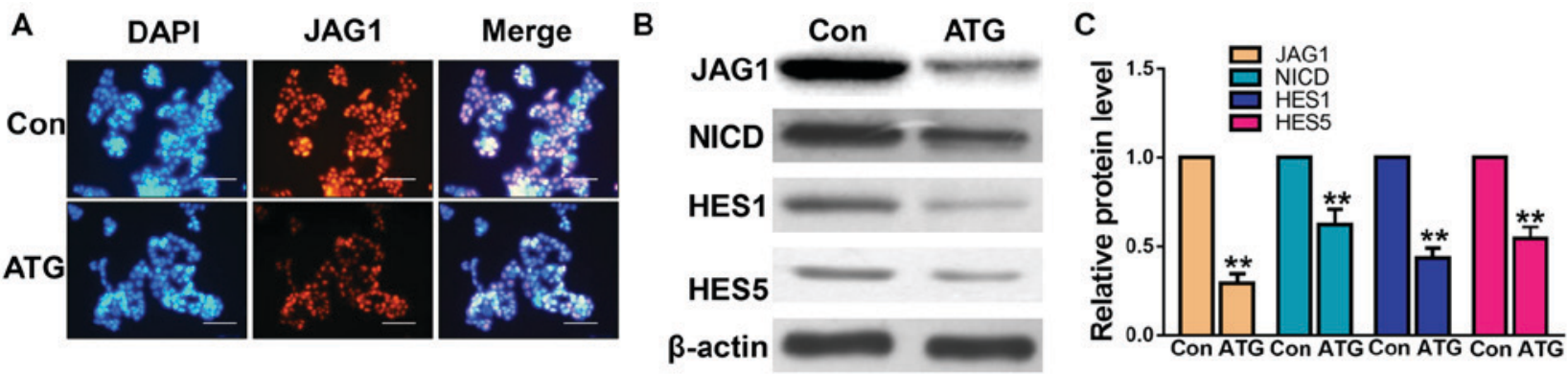

Figure 4. Effects of ATG on the protein expression levels of JAG1, NICD, HES1 and HES5 in the retinoblastoma cell line Y79. (A) Protein expression levels of JAG1 were determined by immunofluorescence assay. (B) Protein expression levels of JAG1, NICD, HES1 and HES5 were analyzed by western blotting. (C) Densitometric analysis. Cells were treated with ATG $(50 \mu \mathrm{M})$ or the equivalent volume of dimethyl sulfoxide. Scale bar, $100 \mu \mathrm{m} .{ }^{* *} \mathrm{P}<0.01 \mathrm{vs}$. Con. ATG, arctigenin; Con, control; HES, transcription factor HES; JAG1, jagged-1; NICD, Notch intracellular domain.

A

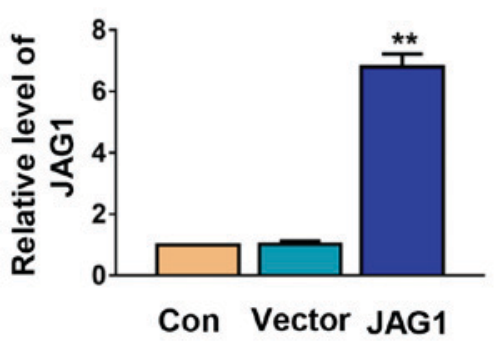

B
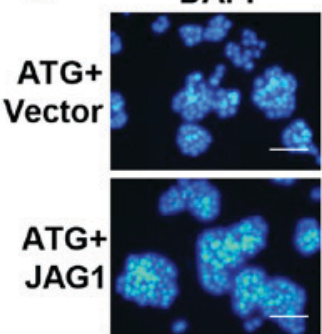

JAG1
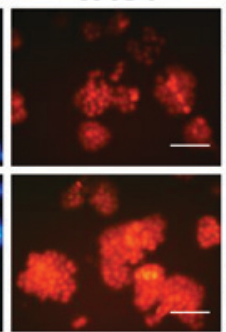

Merge
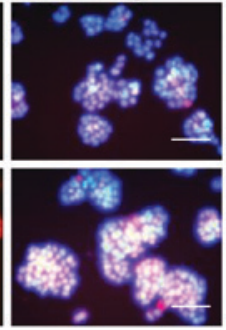
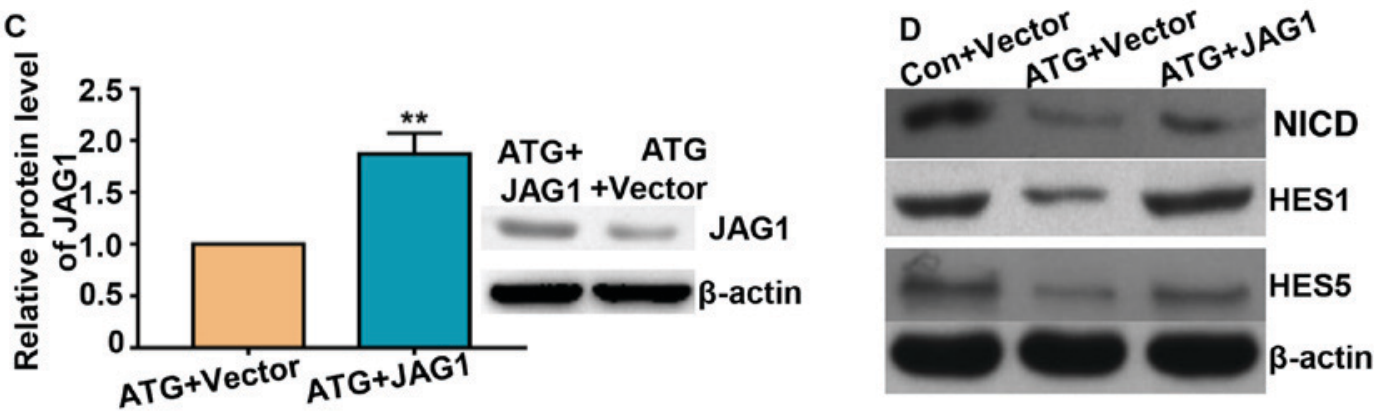

E

$\mathbf{F}$

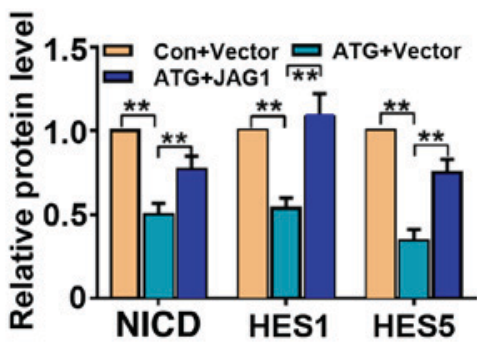

Figure 5. JAG1 mediates the antitumor effects of ATG on the retinoblastoma cell line Y79. (A) JAG1 transfection increased the expression levels of JAG1, as determined by reverse transcription-quantitative polymerase chain reaction analysis. (B) JAG1 overexpression increased the protein expression levels of JAG1 in cells treated with ATG, as determined by immunofluorescence assay. (C) JAG1 overexpression increased the protein expression levels of JAG1 in cells treated with ATG, as determined by western blotting. (D) Protein expression levels of NICD, HES1 and HES5 following treatment with ATG and transfection with JAG1 were determined by western blotting. (E) Densitometric analysis. (F) JAG1 overexpression increased cell viability following treatment with ATG, as determined by Cell Counting kit-8 assay. Cells were transfected with pCDNA3.1(+) vector or pCDNA-JAG1 and were subsequently treated with ATG $(50 \mu \mathrm{M})$ or an equivalent volume of dimethyl sulfoxide. Scale bar, $100 \mu \mathrm{m} .{ }^{* *} \mathrm{P}<0.01$ vs. respective control. ATG, arctigenin; Con, control; HES, transcription factor HES; JAG1, jagged-1; NICD, Notch intracellular domain.

cancer by downregulating the expression levels of epidermal growth factor receptor (11). Additionally, ATG exhibits antimetastatic properties in colorectal cancer cells by modulating the expression of E-cadherin (21) or by suppressing activity of the Wnt/ $\beta$-catenin pathway (8). Similar observations have been made in human lung cancer cells (22). Although the molecular mechanism underlying ATG function has been identified to be associated with various signaling pathways in numerous types 
A
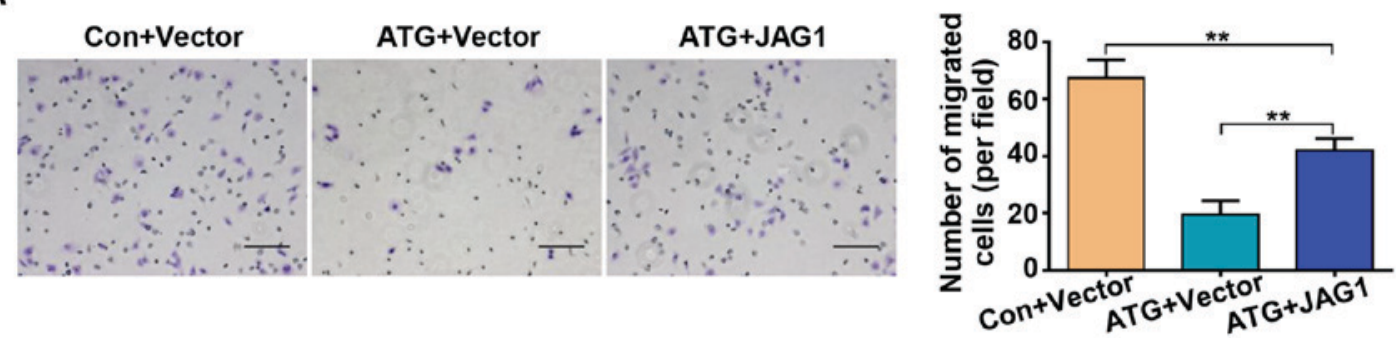

B
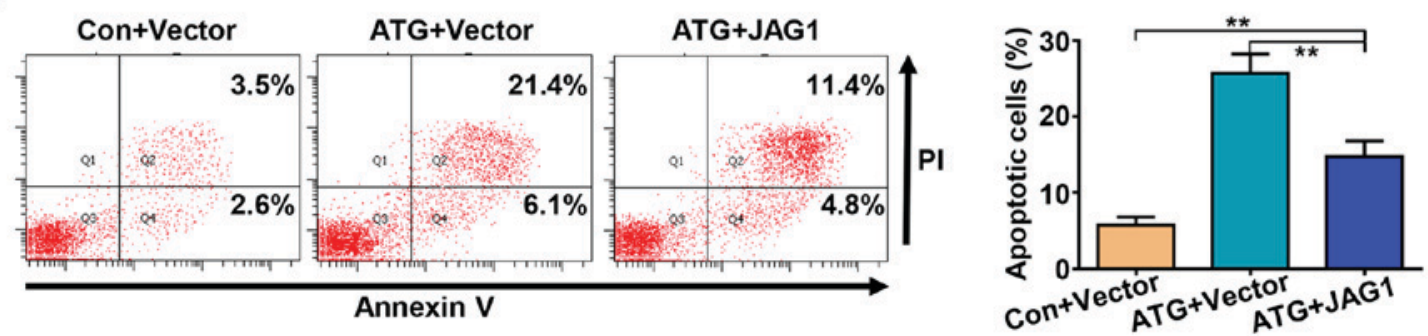

Figure 6. Effects of JAG1 overexpression on the ATG-mediated migration and apoptosis of the retinoblastoma cell line Y79. (A) Effects of JAG1 overexpression on cell migration were determined using Transwell assay. Scale bar, $100 \mu \mathrm{m}$. (B) Effects of JAG1 overexpression on apoptosis were determined by Annexin V/PI staining and quantified by flow cytometry. The cells were transfected with pCDNA3.1(+) vector or pCDNA-JAG1 and were subsequently treated with ATG $(50 \mu \mathrm{M})$ or an equivalent volume of dimethyl sulfoxide. ${ }^{* *} \mathrm{P}<0.01$. ATG, arctigenin; Con, control; JAG1, jagged-1; PI, propidium iodide.

of cancer, whether ATG affects the Notch signaling pathway remains unclear. The present study revealed that treatment with ATG suppressed activity of the Notch signaling pathway by inhibiting the protein expression levels of JAG1.

The Notch signaling pathway is highly conserved among organisms, serving roles in the regulation of cell viability, apoptosis and migration $(12,13,23)$. Notably, the Notch signaling pathway is upregulated in cancer cell lines and tissues, and is able to promote cancer development and progression (15). Conversely, activation of the Notch signaling pathway has been identified to inhibit human tongue carcinoma cells by downregulating the Wnt/ $\beta$-catenin pathway (14) and to suppress the proliferation of small cell lung cancer cells by inducing cell cycle arrest (24). The present results suggested that the Notch signaling pathway may promote the development and progression of retinoblastoma. The present results are consistent with a previous report that demonstrated that microRNA-433 may inhibit retinoblastoma malignancy by suppressing NOTCH1 (25).

In the Notch signaling pathway, the binding between NOTCH and a ligand leads to the release of the NICD from the cell membrane into the nucleus, where the NICD is able to serve as a transcription factor, activating the expression of downstream genes (26). Treatment with ATG decreased the protein expression levels of JAG1 and NICD in this study, indicating a decrease in the activity of the Notch signaling pathway, which led to a decrease in the protein expression levels of the downstream factors HES1 and HES5. In addition to the Notch signaling pathway, treatment with ATG was able to influence the protein expression levels of two factors associated with apoptosis, BCL2 and BAX. Previous studies have investigated the molecular mechanism of ATG, and it has been reported to regulate various signaling pathways, including transforming growth factor $\beta /$ SMAD family member 2/3 (22), p38 mitogen-activated protein kinase (MAPK) (9) and phosphoinositide 3-kinase/AKT serine/threonine kinase 1 (27), suggesting that ATG may serve as an anticarcinogenic factor via a complex regulatory network. Notably, other pathways and molecular factors may be involved, in addition to JAG1 and the Notch signaling pathway, in mediating the effects of ATG in retinoblastoma cells. Furthermore, the Notch signaling pathway was previously identified to undergo crosstalk with multiple signaling pathways, including MAPK (28), Ras (24) and Wnt (29). Therefore, the numerous pathways interacting with the Notch signaling pathway may be involved in the anticarcinogenic effects of ATG. Additionally, JAG1 overexpression may reverse the effects of ATG by influencing multiple pathways associated with the Notch signaling pathway, thus affecting the function of ATG on the regulation of various cellular processes.

In conclusion, the present study suggested that ATG served anticarcinogenic effects on retinoblastoma cells partly by negatively regulating JAG1, indicating that ATG may represent a novel potential therapeutic compound to treat patients with retinoblastoma. Furthermore, the Notch signaling pathway may be further investigated as an additional target to treat retinoblastoma. Nevertheless, further studies are required to examine the multiple roles of ATG and the Notch signaling pathway in retinoblastoma, in order to understand the molecular mechanisms underlying ATG. Notably, only one cell line was investigated in the present study; therefore, the function of ATG in other retinoblastoma cell lines requires further investigation in the future.

\section{Acknowledgements}

Not applicable.

\section{Funding}

The present study was supported by The Traditional Chinese Medicine Project of Chongqing (grant no. 20160322). 


\section{Availability of data and materials}

The datasets used and/or analyzed during the current study are available from the corresponding author on reasonable request.

\section{Authors' contributions}

NK and QL designed the study and performed the experiments. LP analyzed the data and JF, LC and XC helped perform the experiments.

\section{Ethics approval and consent to participate}

Not applicable.

\section{Patient consent for publication}

Not applicable.

\section{Competing interests}

The authors declare that they have no competing interests.

\section{References}

1. Villegas VM, Hess DJ, Wildner A, Gold AS and Murray TG: Retinoblastoma. Curr Opin Ophthalmol 24: 581-588, 2013.

2. Wong FL, Boice JD, Abramson DH, Tarone RE, Kleinerman RA, Stovall M, Goldman MB, Seddon JM, Tarbell N, Fraumeni JF Jr and Li FP: Cancer incidence after retinoblastoma: Radiation dose and sarcoma risk. JAMA 278: 1262-1267, 1997.

3. Luo C and Deng YP: Retinoblastoma: Concerning its initiation and treatment. Int J Ophthalmol 6: 397-401, 2013.

4. Yang C, Fan X and Fan S: Effects and mechanism of puerarin on the human retinoblastoma cells. J Cell Biochem 119: 4506-4513, 2018.

5. Xu Z, Gu C, Wang K, Ju J, Wang H, Ruan K and Feng Y: Arctigenic acid, the key substance responsible for the hypoglycemic activity of Fructus Arctii. Phytomedicine 22: 128-137, 2015.

6. Han YH, Kee JY, Park J, Kim HL, Jeong MY, Kim DS, Jeon YD, Jung Y, Youn DH, Kang J, et al: Arctigenin inhibits adipogenesis by inducing AMPK activation and reduces weight gain in high-fat diet-induced obese mice. J Cell Biochem 117: 2067-2077, 2016.

7. Hyam SR, Lee IA, Gu W, Kim KA, Jeong JJ, Jang SE, Han MJ and Kim DH: Arctigenin ameliorates inflammation in vitro and in vivo by inhibiting the PI3K/AKT pathway and polarizing M1 macrophages to M2-like macrophages. Eur J Pharmacol 708: 21-29, 2013.

8. Zhang S, Li J, Song S, Li J, Tong R, Zang Z, Jiang Q and Cai L: Integrated in silico and experimental methods revealed that Arctigenin inhibited angiogenesis and HCT116 cell migration and invasion through regulating the $\mathrm{H} 1 \mathrm{~F} 4 \mathrm{~A}$ and $\mathrm{Wnt} / \beta$-catenin pathway. Mol Biosyst 11: 2878-2884, 2015.

9. Sihai YU, Yang X: Arctigenin inhibits the proliferation of tongue cancer Tca8113 cells by activation of p38 MAPK. Med J West China 5: 645-647 2015.

10. Wang HQ, Jin JJ and Wang J: Arctigenin enhances chemosensitivity to cisplatin in human nonsmall lung cancer H460 cells through downregulation of survivin expression. J Biochem Mol Toxicol 28: 39-45, 2014.
11. Zhang M, Cai S, Zuo B, Gong W, Tang Z, Zhou D, Weng M, Qin Y, Wang S, Liu J, et al: Arctigenin induced gallbladder cancer senescence through modulating epidermal growth factor receptor pathway. Tumour Biol 39: 1010428317698359, 2017.

12. Qi R, An H, Yu Y, Zhang M, Liu S, Xu H, Guo Z, Cheng T and Cao X: Notch1 signaling inhibits growth of human hepatocellular carcinoma through induction of cell cycle arrest and apoptosis. Cancer Res 63: 8323-8329, 2003.

13. Bolos V, Grego-Bessa J and de la Pompa JL: Notch signaling in development and cancer. Endocr Rev 28: 339-363, 2007.

14. Duan L, Yao J, Wu X and Fan M: Growth suppression induced by Notch1 activation involves Wnt-beta-catenin down-regulation in human tongue carcinoma cells. Biol Cell 98: 479-490, 2006.

15. Sun Y, Zhang R, Zhou S and Ji Y: Overexpression of Notch1 is associated with the progression of cervical cancer. Oncol Lett 9: 2750-2756, 2015 .

16. Sethi N, Dai X, Winter CG and Kang Y: Tumor-derived jagged1 promotes osteolytic bone metastasis of breast cancer by engaging notch signaling in bone cells. Cancer Cell 19: 192-205, 2011.

17. Livak KJ and Schmittgen TD: Analysis of relative gene expression data using real-time quantitative PCR and the 2(-Delta Delta C(T)) method. Methods 25: 402-408, 2001.

18. Sreenivasan S and Krishnakumar S: Synergistic effect of curcumin in combination with anticancer agents in human retinoblastoma cancer cell Lines. Curr Eye Res 40: 1153-1165, 2015.

19. Wang YF, Kunda PE, Lin JW, Wang H, Chen XM, Liu QL and Liu T: Cytokine-induced killer cells co-cultured with complete tumor antigen-loaded dendritic cells, have enhanced selective cytotoxicity on carboplatin-resistant retinoblastoma cells. Oncol Rep 29: 1841-1850, 2013.

20. Sun Y, Tan Y, Lu Z, Li BB, Sun CH, Li T, Zhao LL, Liu Z, Zhang GM, Yao JC and Li J: Arctigenin inhibits liver cancer tumorigenesis by inhibiting gankyrin expression via $\mathrm{C} / \mathrm{EBP} \alpha$ and PPARa. Front Pharmacol 9: 268, 2018.

21. Han YH, Kee JY, Kim DS, Mun JG, Jeong MY, Park SH, Choi BM, Park SJ, Kim HJ, Um JY and Hong SH: Arctigenin inhibits lung metastasis of colorectal cancer by regulating cell viability and metastatic phenotypes. Molecules 21: 1135, 2016.

22. Xu Y, Lou Z and Lee SH: Arctigenin represses TGF- $\beta$-induced epithelial mesenchymal transition in human lung cancer cells. Biochem Biophys Res Commun 493: 934-939, 2017.

23. Yoon $\mathrm{K}$ and Gaiano N: Notch signaling in the mammalian central nervous system: Insights from mouse mutants. Nat Neurosci 8: 709-715, 2005 .

24. Sriuranpong V, Borges MW, Ravi RK, Arnold DR, Nelkin BD, Baylin SB and Ball DW: Notch signaling induces cell cycle arrest in small cell lung cancer cells. Cancer Res 61: 3200-3225, 2001.

25. Li X, Yang L, Shuai T, Piao T and Wang R: MiR-433 inhibits retinoblastoma malignancy by suppressing Notch1 and PAX6 expression. Biomed Pharmacother 82: 247-255, 2016.

26. Penton AL, Leonard LD and Spinner NB: Notch signaling in human development and disease. Semin Cell Dev Biol: 450-457, 2012.

27. Jeong YH, Park JS, Kim DH and Kim HS: Arctigenin increases hemeoxygenase-1 gene expression by modulating PI3K/AKT signaling pathway in rat primary astrocytes. Biomol Ther (Seoul) 22: 497-502, 2014

28. Pear WS, Aster JC, Scott ML, Hasserjian RP, Soffer B, Sklar J and Baltimore D: Exclusive development of T cell neoplasms in mice transplanted with bone marrow expressing activated Notch alleles. J Exp Med 183: 2283-2291, 1996.

29. Guo S, Liu M and Gonzalez-Perez RR: Role of Notch and its oncogenic signaling crosstalk in breast cancer. Biochim Biophys Acta 1815: 197-213, 2011.

This work is licensed under a Creative Commons Attribution-NonCommercial-NoDerivatives 4.0 International (CC BY-NC-ND 4.0) License. 